\title{
Solid Lipid Nanoparticles for Targeted Delivery of Triclosan into Skin for Infection Prevention
}

\author{
Pratibha G. Kakadia, Barbara R. Conway \\ Department of Pharmacy, School of Applied Sciences, University of Huddersfield, Queensgate, \\ Huddersfield \\ HD1 3DH, UK \\ *Corresponding author: b.r.conway@hud.ac.uk
}




\title{
Solid Lipid Nanoparticles for Targeted Delivery of Triclosan into Skin for Infection Prevention
}

\begin{abstract}
Healthcare-associated infections are a concern for health service providers, exacerbated by poor delivery of antimicrobials to target sites within the skin. The dermal route is attractive for local and systemic delivery of drugs however; permeation, penetration and access to deeper skin layers are restricted due to the barrier function of the stratum corneum. Solid lipid nanoparticles present several benefits for topical delivery for therapeutic applications, especially via the follicular route. Hair follicles, surrounded by a close network of blood capillaries and dendritic cells, are an important target for delivery of antimicrobials and present a unique microbial nidus for endogenous infections in situations where the barrier is disrupted, such as after surgery, for example. Triclosan, a broad-spectrum antimicrobial agent, was encapsulated into nanoparticles using glyceryl behenate and glyceryl palmitostearate solid lipids, and incorporating Transcutol P, a known permeation enhancer at different ratios. Optimised formulation were stable over 90 days and in vitro permeation studies using full thickness porcine ear skin showed that the lipid-based nanoparticles enhanced delivery of triclosan into the skin and could direct the agent towards hair follicles, indicating their potential as a carrier system for antiseptic dermal delivery.
\end{abstract}

Keywords: solid lipid nanoparticle, healthcare-associated infection, topical delivery, triclosan, skin antisepsis, follicular delivery 


\section{Introduction}

Healthcare-associated infections (HAIs) are a major concern inflicting significant financial burdens and time constraints on healthcare systems due to increased morbidity and mortality rates, prolonged hospital occupancy and intensified treatment regimes, including repeated surgeries (Vilela et al. 2007). Surgical site infections (SSIs) are a major source of prolonged illness, and a significant cause of death in surgical patients. SSIs occur due to microbial contamination and these microorganisms may originate from either internal or external sources, which includes the patient's own skin, mucous membranes or any contaminated item in the sterile surgical field, including surgical team members, instruments, air, or materials (De Lissovoy et al. 2009). Many aspects of current research to prevent SSIs involve more effective use of antiseptics in order to counteract the world-wide concern of rising antibiotic resistance and the lack of new antibiotic groups entering pharmaceutical development (Leaper et al. 2013). The pilosebaceous units, which are composed of sweat glands (eccrine and apocrine), sebaceous glands and hair follicles, present their own unique nidus and are the most favourable place for microorganism growth due the moist and lipidrich environment (Grice and Segre 2011). However, due to the natural properties of the skin as a protection barrier, there is limited potential for delivery of effective amounts of antimicrobial agents into the skin and this has long been a driver for new strategies to improve penetration of drugs to target microorganisms residing in different areas within the skin (Cevc 1997). Different carrier systems have been developed in an attempt to facilitate the transport of drugs through the skin, enabling drug retention and, in some cases, allowing a controlled release (Kim et al. 2002, Sabu et al. 2018). Amongst these systems, solid lipid nanoparticles (SLNs) are expected to form a depot in the hair follicles, providing targeted controlled drug delivery (Aljuffali et al. 2013). 
SLNs were developed at the beginning of 1990s as an alternative carrier system to emulsions and liposomes by replacing the liquid lipid (oil) of an emulsion by a solid lipid or a blend of solid lipids, i.e. a lipid which is solid at both room and body temperature. SLNs can accommodate both lipophilic and hydrophilic drugs with negligible skin irritation and have improved physical stability and lower cost of production compared to liposomes (Müller, Mäder, Gohla, et al. 2000). Moreover, SLNs have distinct occlusive properties due to the formation of an intact film on the skin surface, which can increase hydration of the stratum corneum (SC) and disrupt packing of corneocytes, thereby facilitating drug penetration into deeper layers of the skin (Jenning and Gohla 2001, Khezri et al. 2018). In addition to the occlusive effect, enhanced drug penetration can also be related to nanometer dimensions of SLNs because the higher surface area facilitates contact of the encapsulated drug with the SC and may favour accumulation for several hours, which allows for sustained drug release (Schäfer-Korting et al., 2007).

TSN (2,4,4-trichloro-hydroxydiphenylether) is a non-cationic, synthetic, broad-spectrum antimicrobial agent used in many soaps, creams, and solutions in concentrations of up to $2 \% \mathrm{w} / \mathrm{w}$ for disinfection of the hands and wounds and for disinfection of the skin before surgery, and in biodegradable sutures. It is used also in oral hygiene products and in preparations for acne and consumer products, such as antibacterial soaps, toothpastes, cosmetics, clothing, kitchenware and toys. Reported minimum inhibitory concentrations (MIC) of TSN for Staphylococcus aureus range between $0.025 \mu \mathrm{g} / \mathrm{ml}$ to $1 \mu \mathrm{g} / \mathrm{ml}$ (Suller and Russell 2000). Polymeric nanoparticles of triclosan (TSN) have been previously evaluated for topical delivery (Domínguez-Delgado et al. 2011, Fagui et al. 2013) with synthesis involving the use of organic solvents, which may cause toxicological issues and irritation. Triclosan has a $\log \mathrm{P}$ of 4.8 and a pKa of 8.1 (Carter et al. 2014) and so has limited local availability within the skin and the hair follicles. 
The aim of our study is to develop SLNs of TSN using a solvent-free method involving hot high shear homogenisation (HSH) followed by probe ultrasonication for delivery to the hair follicles. Glyceryl behenate (GB) and glyceryl palmitostearate (GP) were selected as solid lipids. These lipids are used as thickening agents for topical emulsions and ointments and have been used for SLN formulations previously (Quintanar-Guerrero et al. 2005). Tween ${ }^{\circledR} 80$ (T80) and Transcutol ${ }^{\circledR}$ P (TP) were used as surfactant and cosurfactant respectively. TP has been previously reported to enhance skin penetration for a variety of drugs when used in creams, lotions, microemulsions and aqueous gels (Rowe et al. 2012). Hence the use of TP in SLN formulations is a novel approach to study the increase in skin penetration of TSN.

SLNs were characterised for various physicochemical properties such as particle size, zeta potential, morphology, physical and thermal stability and crystallinity and the formulations evaluated as a topical delivery system using frozen, excised, full thickness porcine ear skin.

\section{Materials and methods}

\subsection{Materials}

Triclosan was obtained from Vivimed (Mumbai, India). Glyceryl behenate, glyceryl palmitostearate and Transcutol ${ }^{\circledR} \mathrm{P}$ were donated by Gattefossé (UK). Tween ${ }^{\circledR} 80$ and sodium lauryl sulphate were purchased from Sigma Aldrich (UK). All other solvents and reagents were of analytical grade.

\subsection{Chromatographic detection of triclosan}

Chromatographic analysis of TSN was performed on a Shimadzu HPLC equipped with a SPD-20 AV Prominence UV/Visible detector set at $280 \mathrm{~nm}$, an LC-20AT pump, and SIL-20A Prominence auto sampler. Separation of TSN was performed using a Kinetex ${ }^{\circledR}$ pentaflurophenyl $5 \mu \mathrm{m}$ column 
$(250 \times 4.6 \mathrm{~mm})\left(\right.$ Phenomenex, UK) maintained at $30^{\circ} \mathrm{C}$ and TSN was eluted with a mobile phase consisting of acetonitrile and water $(60: 40 \mathrm{v} / \mathrm{v})$ at a flow rate of $1 \mathrm{ml} / \mathrm{min}$. Injection volume was 5 $\mu \mathrm{L}$ with retention time of $7.34 \mathrm{~min}$. Standard solutions of TSN were prepared at concentration of $1,5,10,20,30,40$ and $50 \mu \mathrm{g} / \mathrm{ml}$ and these standards were used to determine the linearity $\left(\mathrm{R}^{2}=\right.$ $0.999)$ and the limit of detection $(0.60 \mu \mathrm{g} / \mathrm{ml})$ during HPLC method validation.

\subsection{Preparation of solid lipid nanoparticles}

SLNs were prepared by HSH followed by probe ultrasonication (Kamble et al. 2012, Patel et al. 2012, Nerella et al. 2014, Kaur et al. 2016). Briefly, the lipid phase was prepared by heating lipids (GB and GP) to $5{ }^{\circ} \mathrm{C}$ above their melting points and TSN (equivalent to $10 \mathrm{mg} / \mathrm{g}$ of formulation) was added to the lipids while the aqueous phase was prepared by heating $\mathrm{S}_{\mathrm{mix}}(\mathrm{T} 80$ and TP) and water to same temperature. The hot lipid phase was added slowly to the hot aqueous phase under HSH (Silverson, UK). This hot primary emulsion was then subjected to probe ultrasonication (Sonics and Materials Inc., USA) for $10 \mathrm{~min}$ at $70 \%$ frequency amplitude. The resultant dispersion was cooled to room temperature to solidify the lipids, forming an SLN aqueous dispersion (Mu and Holm 2018). The impact of homogenisation variables on the SLNs was investigated (i.e. homogenisation speed was varied from $4000 \mathrm{rpm}$ to $10,000 \mathrm{rpm}$ and the duration of homogenization ranged from $5 \mathrm{~min}$ to $15 \mathrm{~min}$ ). In addition, the effect of various formulation parameters, such as ratio of surfactant to cosurfactant and lipid to drug ratio, on mean particle size and drug entrapment efficiency were studied. For solid-state characterisation only, SLNs were freeze dried in glass vials under vacuum at atmospheric pressure for $24 \mathrm{~h}$ to obtain a powder and samples were stored at $4^{\circ} \mathrm{C}$ until tested (Christ LD 2-4 Plus, UK).

\subsection{Physicochemical characterisation}

\subsubsection{Particle size analysis}


The particle size and polydispersity index (PDI) of nanoparticles was measured using nanoparticle tracking analysis (NTA, Nanosight LM10, UK). Each sample was suitably diluted with ultrapure water to maintain the concentration of sample between $40-65$ particles/frame and. samples were measured in triplicate to obtain mean particle size and PDI was calculated.

\subsubsection{Zeta potential measurement}

In order to determine the surface charge on nanoparticles, the zeta potential (ZP) was measured using a Zetasizer Nano Z (Malvern Instruments Ltd, UK). Each sample was suitably diluted with filtered, distilled water and placed in a disposable zeta cell. Zeta limit ranged from -200 to +200 $\mathrm{mV}$.

\subsubsection{Determination of drug entrapment efficiency}

The percent drug entrapment efficiency (\% DEE), which corresponds to the percentage of TSN encapsulated within the nanoparticles, was determined by measuring the concentration of free TSN as well as the total concentration of TSN in the dispersion medium. To measure total TSN concentration freeze-dried SLNs were dissolved in a chloroform and methanol $(1: 1 \mathrm{v} / \mathrm{v})$ mixture and sonicated to extract drug from the lipid, while free and unentrapped drug was measured by dissolving the particles in a suitable solvent. Both samples were analysed using the validated HPLC method (section 2.2) and \% DEE was calculated.

\subsubsection{Differential scanning calorimetry}

The thermal behavior of drug and SLNs was analysed by differential scanning calorimetry (DSC) using a Mettler Toledo DSC1 (STAR ${ }^{\mathrm{e}}$ system, UK), calibrated using an indium standard. Samples (8-10 mg) of pure TSN, GB and GP lipids, equivalent ratio of physical mixtures of drug and lipids (PM) and lyophilised TSN-loaded SLNs were analysed in vented $40 \mu \mathrm{l}$ aluminum pans. The 
thermograms were recorded over a temperature range of $25-160^{\circ} \mathrm{C}$ at a heating rate of $10^{\circ} \mathrm{C} / \mathrm{min}$ under a continuous nitrogen gas purge, maintained at a flow rate of $50 \mathrm{ml} / \mathrm{min}$ (Kotikalapudi et al. 2012).

\subsubsection{Powder X-ray diffraction analysis}

The effect of the encapsulation process on the crystallinity of the TSN was investigated using Xray powder diffractometer (XRD) using freeze-dried samples. Powder X-ray diffractogram of the TSN, pure lipid GB and GP, physical mixture of drug and lipids, TSN loaded lipid nanoparticles were analysed using a Bruker diffractometer (Bruker D2 Phase, UK). Samples were placed in a stainless steel holder and the surface powder was levelled manually. Each sample was scanned between 5 and $40^{\circ}$ of $2 \theta$ with step size of $0.019^{\circ}$ and a step time of 32.5 seconds.

\subsubsection{Nanoparticle morphology}

The morphology of SLNs was confirmed using transmission electron microscopy (TEM) using a JEOL 3010, which was operated at $300 \mathrm{kV}$. The SLN suspension was diluted with water and a drop was placed on a 20-mesh carbon coated copper grid (Agar Scientific, UK). Excess sample was removed with filter paper and to achieve effective imaging contrast, the samples were negatively stained with $1 \%(\mathrm{w} / \mathrm{w})$ phosphotungstic acid for $1 \mathrm{~min}$ and air dried at room temperature (Kupetz and Bunjes 2014) .

\subsubsection{Stability study}

Selected SLN formulations stored at room temperature $\left(\sim 20^{\circ} \mathrm{C}\right)$ and in a humidity chamber $\left(40^{\circ} \mathrm{C} / 75 \% \mathrm{RH}\right)$ over a period of three months. The average particle size, PDI and ZP were 
measured at day 30, day 60 and day 90. The samples were studied in triplicate and results were expressed as mean $\pm \mathrm{SD}$.

\subsubsection{Skin permeation study}

\subsubsection{In vitro skin permeation study}

In vitro permeation studies ( $\mathrm{n}=6$ for each formulation and control) were carried out over a period of $24 \mathrm{~h}$ using vertical Franz diffusion cells with a diffusion area of $3.8 \mathrm{~cm}^{2}$ and a receiver compartment volume of $30 \mathrm{ml}$. Porcine skin is reported to be the most appropriate animal model due to the numerous anatomical, histological and physiological similarities with human skin (Dick and Scott 1992, Godin and Touitou 2007, Flaten et al. 2015). Frozen excised full thickness porcine ear skin was used in this study was thawed for $30 \mathrm{~min}$ and then hydrated by immersing in phosphate buffer saline (PBS) pH 7.4 solution for 60 min prior to the start of each experiment. The skin was then cut into appropriate sections and mounted on the Franz diffusion cell, with SC facing the donor compartment (where the formulation was applied) and the dermis facing the receiver compartment. The receiver compartment contained PBS solution ( $\mathrm{pH} 7.4)$ containing $1 \% \mathrm{w} / \mathrm{w}$ SLS $(150 \mathrm{mM})$, in order to ensure skin conditions were maintained throughout the study and water was circulated at $37^{\circ} \mathrm{C}$ to maintain a skin surface temperature at $32^{\circ} \mathrm{C}$, with stirring speed of 200 rpm for $24 \mathrm{~h}$. The skin was equilibrated for $30 \mathrm{~min}$ before loading with SLN formulations (equivalent to $10 \mathrm{mg} / \mathrm{g}$ TSN concentration in an aqueous dispersion) or saturated solution of TSN $(10 \mathrm{mg} / \mathrm{g})$ in $1 \% \mathrm{w} / \mathrm{w}$ SLS as control to each donor compartment and covered with Parafilm to prevent evaporation. Samples $(500 \mu \mathrm{l})$ were withdrawn at regular intervals from receiver compartment (normally 2, 4, 6, 8, 10, 12 and $24 \mathrm{~h}$ ) and replaced by an equal volume of fresh receiver solution to maintain sink conditions. The amount of TSN permeated through skin over 24 h was analysed by HPLC. 


\subsubsection{Quantification of triclosan from skin using differential stripping techniques}

After permeation studies, the skin was removed from diffusion cells and surface of the skin was thoroughly washed with distilled water to remove any excess formulation from the surface and allowed to dry at ambient temperature. A differential stripping technique was used to quantify amount of TSN in porcine skin by combining tape stripping and cyanoacrylate biopsy (Teichmann et al. 2005, Wosika and Cal 2010). The skin was tape-stripped 15 times using an adhesive surgical tape (3M Transpore, UK). Tape 1 was analysed individually as this might contain unabsorbed materials on the skin surface, whereas tapes 2-5 and 6-15 were analyse together to determine the amount of TSN in SC. Following tape stripping, a drop of cyanoacrylate glue was placed on the skin and covered with adhesive tape applied under slight pressure. After 10 min, the cyanoacrylate polymerised and the strip was removed, entrapping the casts of hair follicles. Two successive cyanoacrylate applications were applied to each skin sample and analysed individually.

After stripping, the skin was weighed accurately and cut into fine pieces. All skin samples were placed into vials containing $10 \mathrm{ml}$ of methanol and sonicated for $30 \mathrm{~min}$. After sonication (Transonic, UK), the samples were centrifuged for $20 \mathrm{~min}$ at $400 \mathrm{rpm}$ (Eppendorf centrifuge 5702, UK). The supernatant was collected after centrifugation and the amount of TSN retained in remaining skin tissue was determined using HPLC.

\subsubsection{Statistical analysis}

All particle size, zeta potential and in vitro permeation measurements were performed in triplicate and values were analysed using one way ANOVA (GraphPad Prism 6) with differences considered to be statistically significant when $\mathrm{p}<0.05$.

\section{Results and discussion}


SLNs were formulated and optimised using GB and GP as solid lipids in different concentrations of 3, 5, 7.5 and $10 \%(w / w)$. These lipid carrier systems were stabilised by T80 and TP in different ratios such as 1:1, 2:1, 3:1 and 4:1 respectively. Studying the effect of process and formulation variables on mean particle size and drug entrapment efficiency enabled selection of SLN formulations for further study.

\subsection{Optimisation of process variables}

To optimise homogenisation speed and time, GB-SLNs (GB5-2) and GP-SLNs (GP5-2) were prepared and the effect of different homogenisation speed and time on the mean particle size was determined. It was observed that by increasing homogenising speed from $4000 \mathrm{rpm}$ to $10,000 \mathrm{rpm}$ mean particle size decreased from $520 \pm 0.24 \mathrm{~nm}$ to $193 \pm 0.51 \mathrm{~nm}$ for GB-SLNs, while in case of GP-SLNs mean particle size decreased from $584 \pm 0.47 \mathrm{~nm}$ to $173 \pm 0.36 \mathrm{~nm}$.

The effect of homogenisation time on mean particle size was studied by homogenising SLN formulations for 5, 10 and $15 \mathrm{~min}$. As the duration of homogenisation was extended from 5 to 10 min, the particle size decreased from $426 \pm 0.25 \mathrm{~nm}$ to $217 \pm 0.41 \mathrm{~nm}$ for GB-SLNs and $384 \pm$ $0.29 \mathrm{~nm}$ to $195 \pm 0.37 \mathrm{~nm}$ for GP-SLNs respectively. However, increasing homogenisation time to 15 min shown no further decrease in particle size for both GB-SLN and GP-SLN formulations. As extended exposure of SLNs to excessive cavitation forces may lead to collision and therefore aggregation (Mehnert et al. 2001), SLN formulations were prepared using an optimised homogenisation process at 10,000 rpm for $10 \mathrm{~min}$.

\subsection{Optimisation of formulation variables}

\subsubsection{Effect of lipid and drug ratio}


SLNs were prepared using different concentrations of solid lipid with respect to drug and the impact of lipid concentration on mean particle size and \%DEE was measured (Table 1). It was observed that an increase in lipid concentration with respect to drug, from 3 to $10 \% \mathrm{w} / \mathrm{w}$ for both GB and GP lipids, caused an increase in particle size from $224.15 \pm 5.28 \mathrm{~nm}$ to $839.58 \pm 3.48 \mathrm{~nm}$ and $201.73 \pm 9.23 \mathrm{~nm}$ to $719.31 \pm 6.46 \mathrm{~nm}$ respectively, which may be related to the increased viscosity of the samples. Increase in lipid concentration from 3 to $5 \%(w / w)$, caused an increase in \% DEE for both GB-SLNs and GP-SLNs, but a decrease in \% DEE upon further increases in lipid concentration to 7.5 and $10 \%(\mathrm{w} / \mathrm{w})$.

These changes in DEE may occur due to crystallisation of lipid, which results in expulsion of drug on surface of particles and also high lipid concentration may cause a decrease in diffusion and hence few lipid molecules will be carried into the aqueous phase and therefore reducing entrapment of drug (Abdelbary and Fahmy 2009).

\subsubsection{Effect of surfactant and cosurfactant ratio}

Formation of nanoparticles increases attractive forces between particles due to their small size, which increases surface tension at the interface and leads to physical instability of system. It is important to use an effective concentration of surfactant in the formulation to facilitate particle repulsion and reduce surface tension of the system, however excess surfactant should be avoided to minimise decreases in \% DEE, burst release, toxicity and irritation effects of surfactant. T80 has been used previously as a non-ionic stabiliser for SLN systems (Soares et al. 2013, Shah et al. 2015). It has been reported that a combination of surfactants is more effective for formation of small particles that are stable on storage on, hence TP, a known permeation enhancer, was used as a cosurfactant in SLN formulations (Patel et al. 2010). . 
Increasing the ratio of surfactant and cosurfactant from 1:1 to 4:1 resulted in a corresponding decrease in both particle size as well as \%DEE of SLNs in both GB-SLNs and GP-SLNs (Table 1). A reduction in particle size can be explained by an effective reduction in interfacial tension between aqueous and lipid phases leading to formation of smaller particles and formation of a steric barrier on the particle surface, thereby preventing coalescence (Liu et al. 2007). High surfactant levels in the aqueous phase might increase partitioning of drug from the internal lipid phase to the external phase, decreasing DEE in SLNs (Rahman et al. 2010). Based on the optimisation studies, lipid with concentrations of $3 \%$ and $5 \% \mathrm{w} / \mathrm{w}$, along with Smix (T80: TP) of 2:1, were selected for further study, i.e. GB3-2 and GB5-2 for glyceryl behenate-containing formulations and GP3-2 and GP5-2 for glyceryl palmitostearate-containing formulations.

\subsection{Characterisation of optimised solid lipid nanoparticles}

\subsubsection{Determination of particle size, zeta potential and percent drug entrapment efficiency}

Mean particle size of both lipid formulations ranged from $192.23 \pm 6.39 \mathrm{~nm}$ to $231.72 \pm 4.31 \mathrm{~nm}$. SLNs produced with GB were larger than those containing GP, which may be due to differences in chain lengths and viscosities of lipid used. GB contains glycerol esters of behenic acid $\left(\mathrm{C}_{22}\right)$, where the main fatty acid is behenic acid $>85 \%$ but other fatty acids $\left(\mathrm{C}_{16}-\mathrm{C}_{20}\right)$ are also present. GP is composed of palmitic $\left(\mathrm{C}_{16}\right)$ and stearic acid $\left(\mathrm{C}_{18}\right)>90 \%$. A higher viscosity and longer hydrocarbon chain length of GB might contribute to the larger particle size.

All formulations had a negative $\mathrm{ZP}$, varying from $-20.3 \pm 0.9$ to $-29.9 \pm 2.1 \mathrm{mV}$, indicating relatively good stability and dispersion quality (Essa et al., 2011; Negi et al., 2014). It was also observed that the type of lipid used had no direct relationship with measured zeta potential values. 
$\%$ DEE ranged from $77.17 \pm 0.26$ to $82.19 \pm 1.42$. The \%DEE mainly depends upon the nature of lipids being used as crystalline nature of lipids can lead to lattice formation causing drug expulsion and lipids which are amorphous complex mixtures of triglycerides form less perfect crystals with many imperfections offering more space to accommodate drugs (Müller, Mäder, and Gohla 2000, Tupal et al. 2016). Thus, size, charge and encapsulation efficiency were considered suitable for these to be a potential system for delivery of effective amounts of TSN topically.

\subsubsection{Differential scanning calorimetry}

DSC thermograms for TSN, GB and GP, physical mixtures of drug with both lipids (PM) and TSN loaded SLNs are shown in Figures 1A and B. TSN has a sharp melting endotherm at $61^{\circ} \mathrm{C}$, indicating a degree of crystallinity. The endothermic peak of pure drug was also present in the physical mixture, while pure lipids showed a sharp peak at $76.8^{\circ} \mathrm{C}$ and $57^{\circ} \mathrm{C}$ for GB and GP respectively.

TSN-loaded SLNs exhibited broad endothermic peaks at early temperature point around $64^{\circ} \mathrm{C}$ and $45^{\circ} \mathrm{C}$ for GB and GP. A reduction in endothermic peak temperature of TSN as well as merging of two separate endothermic peaks of TSN and lipids in the SLN formulation generally represents the homogenous dispersion of drug in lipid and nanometric size of the particles (Westesen and Bunjes, 1995, Shah et al., 2015).

\subsubsection{Powder X-ray diffraction analysis}

To study the effect of different glycerides on the crystallinity of TSN in formulation, XRD analysis was performed for pure TSN, GB and GP and TSN-loaded SLNs. TSN is a crystalline material having major diffraction peaks at $2 \theta$ scattered angles 8.2, 24.4 and 25.4 respectively (Celebioglu et al. 2013). The XRD of GB confirms its semi crystalline nature (Figure 2A) with a sharp peak at $2 \theta$ angles of 21.18, 23.38 while GP produces peak at $2 \theta$ angles of 19.5, 21.5 and 23.5 (Figure 
2B). The values are similar to those reported for the lipids in the literature (Bhagwat et al. 2009, Josea et al. 2014). However, a reduction in intensity of TSN peak for both GB-SLNs and GP-SLNs indicates a reduction in crystallinity of TSN, following encapsulation of the drug in the lipid (Jenning and Gohla 2001). Similar results were observed for lipid based nanocarriers of decitabine (Yub et al. 2014) and clotrimazole (Das et al. 2012) and confirm molecular dispersion of the drug in the lipid.

\subsubsection{Nanoparticle morphology}

TEM images (Figure 3A) and particle size data confirmed the spherical nature and nanosized dimensions of the formulations $<250 \mathrm{~nm}$. TEM images (Figure 3B) of SLNs showed a darker central area, which is likely to arise due to drug distribution through the lipid region as proposed following DSC and XRD analysis of formulations.

\subsubsection{Stability studies}

SLN formulations were stored in glass vials and placed at room temperature $\left(20 \pm 2^{\circ} \mathrm{C}\right)$ and controlled humidity conditions $\left(40^{\circ} \mathrm{C} / 75 \% \mathrm{RH}\right)$ to determine stability of formulation in terms of mean particle size, PDI and ZP of SLNs for up to 90 days. There were no statistically significant differences $(\mathrm{p}>0.05)$ in either PDI or ZP for both types of lipid formulations under the different storage conditions (Tables 2 and 3).

\subsubsection{Skin permeation studies}

\subsubsection{In vitro diffusion permeation studies}

Skin permeation studies are relevant while studying topical drug delivery to ensure that formulations display minimum permeation through skin into the receiver medium and maximum 
skin deposition. The influence of type and concentration of lipids and surfactant used, along with other physicochemical parameters on TSN permeation and retention within the skin were evaluated using in vitro skin permeation studies. Skin permeation studies were carried out using excised full thickness porcine ear skin with selected TSN-loaded SLN formulations and saturated aqueous solution of TSN as the control (Figure 4).

SLN formulations had a 4.5-fold higher rate of permeation of TSN compared to a control solution. Similar results were reported for GB-based fluconazole SLNs, which had a 1.73-fold higher fluconazole permeation compared to control solution (Gupta and Vyas, 2012). The increased dermal delivery of drug can be attributed to the structure and physicochemical properties of SLNs. It has been shown that due to their occlusive nature and increased skin hydration of SC, SLNs influence the percutaneous permeation of drugs present in the formulations (Gupta and Vyas, 2012; Schäfer-Korting et al., 2007). Increasing lipid concentrations to 5\% w/w (GB5-2 and GP5-

2) resulted in a 2-fold increase in TSN permeation compared to GB3-2 and GP3-2 formulations respectively, which might be due to smaller particle size, higher \% DEE and a more occlusive effect due to lipid content. In this study, the type and concentration of lipid affected the flux of TSN as GP5-2 formulation exhibited the highest diffusion rate through skin compared to other formulations and the control $(\mathrm{p}<0.002)$ (Table 4).

\subsubsection{Quantification of triclosan in the skin}

The amount of TSN retained within skin was determined by adhesive tape stripping and cyanoacrylate biopsies. A differential stripping technique was used to quantify TSN levels in different areas of the skin, including hair follicles, epidermis and dermis and the results are presented in Figure 5. SLNs significantly increased TSN retention within the skin compared to the 
control solution, $(\mathrm{p}<0.05)$. Epidermal targeting by SLNs was proposed to be due to occlusion and interaction of SLNs with skin lipids (Chen et al. 2006). GP-based SLNs delivered more TSN into the skin and hair follicles compared to GB-based SLNs.

The amount of TSN recovered from skin appendages through cyanoacrylate biopsy was dependent on the concentration of lipid used in the formulations. As lipid concentration increased from $3 \%$ to $5 \%(\mathrm{w} / \mathrm{w})$ in GB-based SLNs, there was a 2-fold increase in TSN concentration from $0.28 \pm$ $0.07 \mu \mathrm{g} / \mathrm{mg}$ to $0.57 \pm 0.08 \mu \mathrm{g} / \mathrm{mg}$ while for GP-based SLNs, the concentration of TSN increased from $0.97 \pm 0.04 \mu \mathrm{g} / \mathrm{mg}$ to $1.99 \pm 0.14 \mu \mathrm{g} / \mathrm{mg}$ in the appendages. The application of the GP5-2 SLN formulation increased TSN retention in skin and in the follicles, most likely due to their small size and high encapsulation efficiency. Thus, this deeper penetration of drug into skin and targeted delivery of follicles render it a promising formulation for effective antiseptic delivery into the skin.

\section{Conclusion}

This study demonstrates the potential of a lipid-based formulation to increase the dermal absorption of TSN. Biodegradable lipids GB and GP along with a mixture of surfactant, T80 and TP were used to produce stable SLNs. Various process and formulation parameters were analysed to optimise SLNs for skin delivery. In vitro permeation studies showed GP formulations containing lipid-to-drug ratio of 5:1 preferentially targeted the follicular route compared to both GB formulation and control solution. Using SLNs to target antiseptic agents to the skin appendages has potential to enhance and sustain activity to improve skin antisepsis, a major goal in the reduction of healthcare-associated infections. 
We would like to thank Dr Jeremy Hopwood for TEM images, Gattefossé for the samples and the University of Huddersfield for funding the project.

Figure Legends

Figure 1. (A) DSC thermograms of triclosan (TSN) and lipid (GB), physical mixture (PM) of GB and TSN, TSN loaded GB-SLNs (GB3-2, GB5-2), (B) DSC thermograms of TSN, lipid (GP), physical mixture (PM) of GP and TSN, TSN loaded GP-SLNs (GP3-2, GP5-2).

Figure 2. (A) XRD powder diffractograms of triclosan (TSN), lipid (GB) andloaded nanoparticles (GB3-2, GB5-2), (B) XRD powder diffractograms of triclosan (TSN) and lipid, (GP), and loaded nanoparticles (GP3-2, GP5-2).

Figure 3 A and B. TEM images of triclosan loaded SLNs (1000 x).

Figure 4. In vitro cumulative amount of triclosan permeated following $24 \mathrm{~h}$ topical application of SLN formulations and control solution through porcine ear skin (Mean $\pm \mathrm{SD}, \mathrm{n}=6)$.

Figure 5. Triclosan uptake into skin treated with SLN formulations and control solution (Mean \pm $\mathrm{SD}, \mathrm{n}=6)$. 
References:

Abdelbary, G. and Fahmy, R.H., 2009. Diazepam loaded solid lipid nanoparticles: Design and Characterisation. AAPS PharmSciTech, 10, 211-219.

Aljuffali, I.A., Sung, C.T., Shen, F.M., Huang, C.T., and Fang, J.Y., 2013. Squarticles as a lipid nanocarrier for delivering diphencyprone and minoxidil to hair follicles and human dermal papilla cells. The AAPS Journal, 16 (1), 140-150.

Bhagwat, D.A., Kawtikwar, P.S., and Sakarkar, D.M., 2009. Formulation and the in vitro and biopharmaceutical evaluation of sustained release tablet of verapamil $\mathrm{HCl}$ using Precirol ATO 5 through melt granulation technique. Asian Journal of Pharmaceutics, 3 (4), 278285.

Carter, L., Eleanor, H., Williams, M., Ryan, J., Kookana, R., and Boxall, A., 2014. Fate and Uptake of Pharmaceuticals in Soil-Plant Systems. Journal of Agricultural and Food Chemistry, 62, 816-825.

Celebioglu, A., Umu, O.C., Tekinay, T., and Uyar, T., 2013. Antibacterial electrospun nanofibers from triclosan/cyclodextrin inclusion complexes. Colloids and Surfaces B: Biointerfaces, $116,612-619$.

Cevc, G., 1997. Drug delivery across the skin. Expert opinion on Investigational drugs, 6, 18871937.

Chen, H., Chang, X., Du, D., Liu, W., Liu, J., Weng, T., Yang, Y., Xu, H., and Yang, X., 2006. Podophyllotoxin loaded solid lipid nanoparticles for epidermal targeting. Journal of Controlled Release, 110, 296-306.

Das, S., Ng, W.K., and Tan, R.B.H., 2012. Are nanostructured lipid carriers (NLCs) better than solid lipid nanoparticles (SLNs): Development, characterisations and comparative evaluations of clotrimazole loaded SLNs and NLCs? European Journal of Pharmaceutical Sciences, 47 (1), 139-151.

Dick, I.P. and Scott, R.C., 1992. Pig ear skin as an in vitro model for human permeability. Journal of Pharmaceutics and Pharmacology, 44, 640-645.

Domínguez-Delgado, C.L., Rodríguez-Cruz, I.M., Escobar-Chávez, J.J., Calderón-Lojero, I.O., 
Quintanar-Guerrero, D., and Ganem, A., 2011. Preparation and characterisation of triclosan nanoparticles intended to be used for the treatment of acne. European journal of Pharmaceutics and Biopharmaceutics, 79 (1), 102-117.

Essa, S., Rabanel, J.M., and Hildgen, P., 2011. Characterisation of rhodamine loaded PEG-gPLA nanoparticles (NPs): Effect of poly(ethyleneglycol) grafting density. International Journal of Pharmaceutics, 411, 178-187.

Fagui, A., Dubot, P., Loftsson, T., and Amiel, C., 2013. Triclosan loaded with high encapsulation efficiency into PLA nanoparticles coated with? cyclodextrin polymer. Journal of Inclusion Phenomena and Macrocyclic Chemistry, 75 (3-4), 277-283.

Flaten, G.E., Palac, Z., Engesland, A., Filipović-Grčić, J., Vanić, Ž., and Škalko-Basnet, N., 2015. In vitro skin models as a tool in optimisation of drug formulation. European Journal of Pharmaceutical Sciences, 75, 10-24.

Godin, B. and Touitou, E., 2007. Transdermal skin delivery: Predictions for human from in vivo, ex vivo and animal models. Advanced Drug Delivery Reviews, 59, 1152-1161.

Grice, E.A. and Segre, J.A., 2011. The skin microbiome. Nature Reviews Microbiology, 9 (4), 244-253.

Gupta, M. and Vyas, S.P., 2012a. Development, characterisation and in vivo assessment of effective lipidic nanoparticles for dermal delivery of fluconazole against cutaneous candidiasis. Chemistry and Physics of Lipids, 165 (4), 454-461.

Gupta, M. and Vyas, S.P., 2012b. Development, characterisation and in vivo assessment of effective lipidic nanoparticles for dermal delivery of fluconazole against cutaneous candidiasis. Chemistry and Physics of Lipids, 165, 454-461.

Jenning, V. and Gohla, S., 2001. Encapsulation of retinoids in solid lipid nanoparticles. Journal of Microencapsulation, 18, 149-158.

Josea, S., Anjua, S.S., Cinua, T.A., Aleykuttya, N.A., Thomasc, S., and Souto, E.B., 2014. In vivo pharmacokinetics and biodistribution of resveratrol-loaded solid lipid nanoparticles for brain delivery. International Journal of Pharmaceutics, 20 (1-2), 6-13.

Kamble, M.S., Vaidya, K.K., Bhosale, A. V, and Chaudhari, P.D., 2012. Solid lipid nanoparticles and Nanostructured lipid carriers - An overview. International Journal of 
Pharmaceutical, Chemical and Biological Sciences, 2 (4), 681-691.

Kaur, A., Goindi, S., and Katare, O., 2016. Formulation, characterisation and in vivo evaluation of lipid based nanocarriers for topical delivery of diflunisal. Journal of Microencapsulation, 33 (5), 475-486.

Khezri, K., Dizaj, M.S., and Maleki, S., 2018. Application of nanoparticles in percutaneous delivery of active ingredients in cosmetic preparations. Biomedicine and Pharmacotherapy, 106, 1499-1505.

Kim, J.C., Song, M., Kim, M., Lee, E., Park, S., Rang, M., and Ahn, H., 2002. Preparation and characterisation of triclosan containing vesicles. Colloids and Surfaces B: Biointerfaces, 26 (3), 235-241.

Kotikalapudi, L.S., Adepu, L., VijayaRatna, J., and Diwan, P. V, 2012. Formulation and in vitro characterisation of domperidone loaded solid lipid nanoparticles. International Journal of Pharmaceutical and Biomedical Research, 3 (1), 22-29.

Kupetz, E. and Bunjes, H., 2014. Lipid nanoparticles: Drug localisation is substance specific and achievable load depends on the size and physical state of the particles. Journal of Controlled Release, 189, 54-64.

Leaper, D., Fry, D., and Assadian, O., 2013. Perspectives in prevention and treatment of surgical site infection - a narrative review of the literature. Wounds, 25 (11), 313-323.

De Lissovoy, G., Fraeman, K., Hutchins, V., Murphy, D., Song, D., and Vaughn, B.B., 2009. Surgical site infection: Incidence and impact on hospital utilisation and treatment costs. American Journal of Infection Control, 37 (5), 387-397.

Liu, J., Gong, T., Wang, C., Zhong, Z., and Zhang, Z., 2007. Solid lipid nanoparticles loaded with insulin by sodium cholate phosphatidylcholine based mixed micelles: Preparation and characterisation. International Journal of Pharmaceutics, 340, 153-162.

Mehnert, W., Mäder, K., and Wolfgang, M., 2001. Solid lipid nanoparticles production, characterisation and applications. Advanced Drug Delivery Reviews, 47 (2-3), 83-101.

Mu, H. and Holm, R., 2018. Solid lipid nanocarriers in drug delivery: characterization and design. Expert Opinion on Drug Delivery, 15, 771-785. 
Müller, R.H., Mäder, K., and Gohla, S.H., 2000. Solid lipid nanoparticles for controlled drug delivery: A review of the state of the art. European Journal of Pharmaceutics and Biopharmaceutics, 50 (1), 161-177.

Müller, R.H., Mäder, K., Gohla, S.H., Muller, R.H., and Mader, K., 2000. Solid lipid nanoparticles for controlled drug delivery: A review of the state of the art. European Journal of Pharmaceutics and Biopharmaceutics, 50 (1), 161-177.

Negi, J.S., Chattopadhyay, P., Sharma, A.K., and Ram, V., 2014a. Development and evaluation of glyceryl behenate based solid lipid nanoparticles (SLNs) using hot self nanoemulsification (SNE) technique. Archives of Pharmacal Research, 37 (3), 361-370.

Negi, J.S., Chattopadhyay, P., Sharma, A.K., and Ram, V., 2014b. Development and evaluation of glyceryl behenate based solid lipid nanoparticles (SLNs) using hot selfnanoemulsification (SNE) technique. Archives of Pharmacal Research, 37 (3), 361-370.

Nerella, A., Basava, R., and Devi, A., 2014. Formulation, optimisation and in vitro characterisation of letrozole loaded solid lipid nanoparticles. International Journal of Pharmaceutical Sciences and Drug Research, 6 (3), 183-188.

Patel, D., Dasgupta, S., Dey, S., Ramani, Y.R., Ray, S., and Mazumder, B., 2012. Nanostructured lipid carriers (NLC) based gel for the topical delivery of aceclofenac: Preparation, characterisation, and in vivo evaluation. Scientia Pharmaceutica, 80 (3), 749764.

Patel, M.J., Patel, N.M., Patel, R.B., and Patel, R.P., 2010. Formulation and evaluation of self microemulsifying drug delivery system of lovastatin. Asian Journal of Pharmaceutical Sciences, 5 (6), 266-275.

Quintanar-Guerrero, D., Tamayo-Esquivel, D., Ganem-Quintanar, A., Allémann, E., and Doelker, E., 2005. Adaptation and optimization of the emulsification-diffusion technique to prepare lipidic nanospheres. European Journal of Pharmaceutical Sciences, 26 (2), 211218.

Rahman, Z., Zidan, A.S., and Khan, M.A., 2010. Non destructive methods of charcaterisation of risperidone solid lipid nanoparticles. European Journal of Pharmaceutics and Biopharmaceutics, 76, 127-137. 
Rowe, R.C., Sheskey, P.J., Cook, W.G., and Fenton, M.E., 2012. Diethylene glycol monoethyl ether. In: Handbook of Pharmaceutical Excipients. Washington, DC, 256-258.

Sabu, C., Rejo, C., Kotta, S., and Pramod, K., 2018. Bioinspired and biomimetic systems for advanced drug and gene delivery. Journal of Controlled Release, 287 (10), 142-155.

Schäfer-Korting, M., Wolfgang, M., and Hans-Christian, K., 2007. Lipid nanoparticles for improved topical application of drugs for skin diseases. Advanced Drug Delivery Reviews, $59,427-443$.

Schäfer-Korting, M., Wolfgang, M., and Korting, H., 2007. Lipid nanoparticles for improved topical application of drugs for skin diseases. Advanced Drug Delivery Reviews, 59, $427-$ 443.

Shah, B., Khunt, D., Bhatt, H., Misra, M., and Padh, H., 2015. Application of quality by design approach for intranasal delivery of rivastigmine loaded solid lipid nanoparticles: Effect on formulation and characterisation parameters. European Journal of Pharmaceutical Sciences, 78, 54-66.

Soares, S., Fonte, P., Costa, A., Andrade, J., Seabra, V., Ferreira, D., Reis, S., and Sarmento, B., 2013. Effect of freeze drying, cryoprotectants and storage conditions on the stability of secondary structure of insulin-loaded solid lipid nanoparticles. International Journal of Pharmaceutics, 456 (2), 370-381.

Suller, M.T. and Russell, a D., 2000. Triclosan and antibiotic resistance in Staphylococcus aureus. The Journal of Antimicrobial Chemotherapy, 46, 11-18.

Teichmann, A., Jacobi, U., Ossadnik, M., Richter, H., Koch, S., Sterry, W., and Lademann, J., 2005. Differential stripping: Determination of the amount of topically applied substances penetrated into the hair follicles. Journal of Investigative Dermatology, 125, 264-269.

Tupal, A., Sabzichi, M., Ramezani, F., Kouhsoltani, M., and Hamishehkar, H., 2016. Dermal delivery of doxorubicin-loaded solid lipid nanoparticles for the treatment of skin cancer. Journal of Microencapsulation: Micro and Nano Carriers, 33 (4), 372-380.

Vilela, R., Jacomo, A.D.N., and Tresoldi, A.T., 2007. Risk factors for central venous catheterrelated infections in paediatric intensive care. Clinical Science, 62 (5), 537.544.

Wosika, H. and Cal, K., 2010. Targeting to hair follicles: Current status and potential. Journal of 
Dermatological Science, 57, 83-89.

Yub, R., Srivastava, M., Ahmad, N., Kumar, N., Bhatnagar, A., and Kohli, K., 2014. Lipid based nanocarrier system for the potential oral delivery of decitabine: Formulation design, characterisation, ex vivo, and in vivo assessment. International Journal of Pharmaceutics, 477, 601-612. 
Table 1. Effect of concentration of lipids and surfactants on particle size and percent entrapment efficiency of SLNs (Mean $\pm \mathrm{SD}, \mathrm{n}=3$ ).

\begin{tabular}{|c|c|c|c|c|c|c|c|c|c|}
\hline \multirow{2}{*}{$\begin{array}{l}\text { Lipid: } \\
\text { drug } \\
\text { ratio }\end{array}$} & \multicolumn{2}{|c|}{ Particle Size $(\mathrm{nm})$} & \multicolumn{2}{|c|}{$\% \mathrm{DEE}$} & \multirow{2}{*}{$\begin{array}{r}\text { T80: } \\
\text { TP } \\
\text { ratio }\end{array}$} & \multicolumn{2}{|c|}{ Particle Size $(\mathrm{nm})$} & \multicolumn{2}{|c|}{$\%$ DEE } \\
\hline & GB-SLN & GP-SLN & GB-SLN & GP-SLN & & GB-SLNs & GP-SLN & GB-SLN & GP-SLN \\
\hline $3: 1$ & $\begin{array}{c}224.15 \pm \\
5.28\end{array}$ & $\begin{array}{c}201.73 \pm \\
9.23\end{array}$ & $76.2 \pm 1.63$ & $\begin{array}{l}79.3 \pm \\
1.86\end{array}$ & $1: 1$ & $\begin{array}{l}315 \pm \\
1.33\end{array}$ & $\begin{array}{l}286 \pm \\
1.27\end{array}$ & $\begin{array}{c}81.3 \pm \\
0.79\end{array}$ & $\begin{array}{c}84.5 \pm \\
1.53\end{array}$ \\
\hline 5: 1 & $\begin{array}{c}231.72 \pm \\
6.52\end{array}$ & $\begin{array}{c}192.23 \pm \\
6.29\end{array}$ & $83.6 \pm 2.47$ & $\begin{array}{c}87.2 \pm \\
1.48\end{array}$ & 2: 1 & $\begin{array}{l}217 \pm \\
2.12\end{array}$ & $\begin{array}{l}184 \pm \\
1.78\end{array}$ & $\begin{array}{c}79.6 \pm \\
1.38\end{array}$ & $\begin{array}{c}81.7 \pm \\
1.04\end{array}$ \\
\hline 7.5: 1 & $\begin{array}{c}683.91 \pm \\
8.69\end{array}$ & $\begin{array}{c}597.39 \pm \\
8.52\end{array}$ & $77.5 \pm 1.06$ & $\begin{array}{c}75.1 \pm \\
1.94\end{array}$ & $3: 1$ & $\begin{array}{l}196 \pm \\
0.93\end{array}$ & $\begin{array}{l}171 \pm \\
2.04\end{array}$ & $\begin{array}{c}68.4 \pm \\
1.73\end{array}$ & $\begin{array}{l}73.5 \pm \\
2.18\end{array}$ \\
\hline 10: 1 & $\begin{array}{c}839.59 \pm \\
3.48\end{array}$ & $\begin{array}{c}719.31 \pm \\
6.46\end{array}$ & $\begin{array}{c}68.23 \pm \\
2.18\end{array}$ & $65.8 \pm 2.4$ & 4: 1 & $\begin{array}{l}163 \pm \\
1.64\end{array}$ & $\begin{array}{l}154 \pm \\
2.36\end{array}$ & $\begin{array}{l}60.1 \pm \\
1.28\end{array}$ & $\begin{array}{c}66.2 \pm \\
1.57\end{array}$ \\
\hline
\end{tabular}

$\%$ DEE-Drug encapsulation efficiency

GB-glyceryl behenate

GP-gylceryl palmitostearate

T80-Tween 80

TP-Transcutol P

SLN-solid lipid nanoparticle 
Table 2. Particle size, polydispersity index (PDI) and zeta potential (ZP) of SLN formulations stored at room temperature $20 \pm 2^{\circ} \mathrm{C}$. (Mean $\pm \mathrm{SD}, \mathrm{n}=3$ ).

\begin{tabular}{|c|c|c|c|c|}
\hline Duration & Formulations & Particle size $(\mathrm{nm})$ & PDI & $\mathrm{ZP}(\mathrm{mV})$ \\
\hline \multirow[t]{4}{*}{ Day 0} & GB3-2 & $175.2 \pm 9.2$ & $0.86 \pm 0.03$ & $-20.3 \pm 0.9$ \\
\hline & GB5-2 & $180.1 \pm 7.3$ & $0.89 \pm 0.01$ & $-25.9 \pm 0.6$ \\
\hline & GP3-2 & $193.8 \pm 8.3$ & $0.62 \pm 0.05$ & $-24.4 \pm 0.8$ \\
\hline & GP5-2 & $169.3 \pm 6.2$ & $0.87 \pm 0.04$ & $-29.9 \pm 1.2$ \\
\hline \multirow[t]{4}{*}{ Day 30} & GB3-2 & $175.3 \pm 10.6$ & $0.86 \pm 0.02$ & $-25.3 \pm 0.6$ \\
\hline & GB5-2 & $188.2 \pm 8.4$ & $0.61 \pm 0.07$ & $-29.1 \pm 0.5$ \\
\hline & GP3-2 & $194.3 \pm 9.5$ & $0.73 \pm 0.03$ & $-29.0 \pm 0.4$ \\
\hline & GP5-2 & $170.6 \pm 7.3$ & $0.89 \pm 0.08$ & $-30.3 \pm 0.9$ \\
\hline \multirow[t]{4}{*}{ Day 60} & GB3-2 & $180.2 \pm 10.9$ & $0.92 \pm 0.01$ & $-24.6 \pm 0.5$ \\
\hline & GB5-2 & $203.8 \pm 9.3$ & $0.68 \pm 0.04$ & $-26.5 \pm 0.4$ \\
\hline & GP3-2 & $198.2 \pm 9.8$ & $0.83 \pm 0.08$ & $-32.7 \pm 0.7$ \\
\hline & GP5-2 & $179.6 \pm 8.1$ & $0.96 \pm 0.09$ & $-29.4 \pm 0.9$ \\
\hline \multirow[t]{4}{*}{ Day 90} & GB3-2 & $188.9 \pm 11.2$ & $0.93 \pm 0.03$ & $-28.8 \pm 0.8$ \\
\hline & GB5-2 & $209.6 \pm 9.7$ & $0.72 \pm 0.07$ & $-25.5 \pm 0.3$ \\
\hline & GP3-2 & $216.2 \pm 10.3$ & $0.86 \pm 0.04$ & $-22.6 \pm 0.6$ \\
\hline & GP5-2 & $184.7 \pm 8.6$ & $0.98 \pm 0.06$ & $-28.7 \pm 0.8$ \\
\hline
\end{tabular}

GB3-2-solid lipid nanoparticles comprising glyceryl behenate $3 \% \mathrm{w} / \mathrm{w}$ and with a surfactant/cosurfactant ratio of 2:1 GB5-2-solid lipid nanoparticles comprising glyceryl behenate $5 \% \mathrm{w} / \mathrm{w}$ and with a surfactant/cosurfactant ratio of 2:1 GP3-2-solid lipid nanoparticles comprising glyceryl palmitostearate $3 \% \mathrm{w} / \mathrm{w}$ and with a surfactant/cosurfactant ratio of $2: 1$

GP3-2-solid lipid nanoparticles comprising glyceryl palmitostearate $5 \% \mathrm{w} / \mathrm{w}$ and with a surfactant/cosurfactant ratio of $2: 1$ 
Table 3. Particle size, polydispersity index (PDI) and zeta potential (ZP) of SLN formulations stored under controlled humidity at $40^{\circ} \mathrm{C} / 75 \% \mathrm{HR}$. (Mean $\pm \mathrm{SD}, \mathrm{n}=3$ ).

\begin{tabular}{|c|c|c|c|c|}
\hline Duration & Formulations & $\begin{array}{l}\text { Particle size } \\
(\mathrm{nm})\end{array}$ & PDI & $\mathrm{ZP}(\mathrm{mV})$ \\
\hline \multirow[t]{4}{*}{ Day 0} & GB3-2 & $175.2 \pm 9.2$ & $0.86 \pm 0.03$ & $-20.3 \pm 0.9$ \\
\hline & GB5-2 & $180.1 \pm 7.3$ & $0.89 \pm 0.01$ & $-25.9 \pm 0.6$ \\
\hline & GP3-2 & $193.8 \pm 8.3$ & $0.62 \pm 0.05$ & $-24.4 \pm 0.8$ \\
\hline & GP5-2 & $169.3 \pm 6.2$ & $0.87 \pm 0.04$ & $-29.9 \pm 1.2$ \\
\hline \multirow[t]{4}{*}{ Day 30} & GB3-2 & $180.3 \pm 10.2$ & $0.93 \pm 0.02$ & $-26.8 \pm 1.2$ \\
\hline & GB5-2 & $195.6 \pm 7.9$ & $0.95 \pm 0.04$ & $-30.2 \pm 1.5$ \\
\hline & GP3-2 & $200.3 \pm 8.4$ & $0.74 \pm 0.05$ & $-29.9 \pm 1.3$ \\
\hline & GP5-2 & $168.9 \pm 6.9$ & $0.85 \pm 0.03$ & $-33.8 \pm 1.8$ \\
\hline \multirow[t]{4}{*}{ Day 60} & GB3-2 & $208.5 \pm 11.4$ & $1.02 \pm 0.08$ & $-29.4 \pm 1.6$ \\
\hline & GB5-2 & $220.6 \pm 8.3$ & $1.31 \pm 0.04$ & $-32.4 \pm 1.8$ \\
\hline & GP3-2 & $203.2 \pm 8.6$ & $1.24 \pm 0.03$ & $-31.7 \pm 1.9$ \\
\hline & GP5-2 & $174.8 \pm 7.2$ & $1.06 \pm 0.06$ & $-34.9 \pm 2.1$ \\
\hline \multirow[t]{4}{*}{ Day 90} & GB3-2 & $217.4 \pm 11.6$ & $1.34 \pm 0.01$ & $-32.8 \pm 1.9$ \\
\hline & GB5-2 & $228.1 \pm 9.2$ & $1.45 \pm 0.04$ & $-33.5 \pm 2.3$ \\
\hline & GP3-2 & $210.5 \pm 9.1$ & $1.30 \pm 0.07$ & $-32.4 \pm 2.5$ \\
\hline & GP5-2 & $209.7 \pm 7.8$ & $1.25 \pm 0.05$ & $-34.9 \pm 2.7$ \\
\hline
\end{tabular}

GB3-2-solid lipid nanoparticles comprising glyceryl behenate $3 \% \mathrm{w} / \mathrm{w}$ and with a surfactant/cosurfactant ratio of 2:1 GB5-2-solid lipid nanoparticles comprising glyceryl behenate $5 \% \mathrm{w} / \mathrm{w}$ and with a surfactant/cosurfactant ratio of 2:1 GP3-2-solid lipid nanoparticles comprising glyceryl palmitostearate $3 \% \mathrm{w} / \mathrm{w}$ and with a surfactant/cosurfactant ratio of $2: 1$

GP3-2-solid lipid nanoparticles comprising glyceryl palmitostearate $5 \% \mathrm{w} / \mathrm{w}$ and with a surfactant/cosurfactant ratio of $2: 1$ 
Table 4. In vitro permeability parameters of TSN-loaded SLN formulations and control solution in porcine ear skin (Mean $\pm S D, n=6$ ).

\begin{tabular}{|c|c|c|}
\hline Formulation & $\begin{array}{c}\text { Flux }\left(\mathrm{J}_{\mathrm{ss}}\right) \\
\mu \mathrm{g} / \mathrm{cm}^{2} / \mathrm{h}\end{array}$ & $\begin{array}{c}\text { Permeability coefficient } \\
\left(\mathrm{K}_{\mathrm{p}}\right) \times 10^{-5} \mathrm{~cm} / \mathrm{h}\end{array}$ \\
\hline Control & $0.086 \pm 0.05$ & $0.86 \pm 0.13$ \\
\hline GB3-2 & $0.202 \pm 0.07$ & $2.02 \pm 0.06$ \\
\hline GB5-2 & $0.245 \pm 0.03$ & $2.45 \pm 0.12$ \\
\hline GP3-2 & $0.279 \pm 0.04$ & $2.79 \pm 0.16$ \\
\hline GP5-2 & $0.536 \pm 0.06$ & $5.36 \pm 0.38$ \\
\hline
\end{tabular}

GB3-2-solid lipid nanoparticles comprising glyceryl behenate $3 \% \mathrm{w} / \mathrm{w}$ and with a surfactant/cosurfactant ratio of 2:1 GB5-2-solid lipid nanoparticles comprising glyceryl behenate $5 \% \mathrm{w} / \mathrm{w}$ and with a surfactant/cosurfactant ratio of 2:1 GP3-2-solid lipid nanoparticles comprising glyceryl palmitostearate $3 \% \mathrm{w} / \mathrm{w}$ and with a surfactant/cosurfactant ratio of $2: 1$

GP3-2-solid lipid nanoparticles comprising glyceryl palmitostearate $5 \% \mathrm{w} / \mathrm{w}$ and with a surfactant/cosurfactant ratio of $2: 1$ 

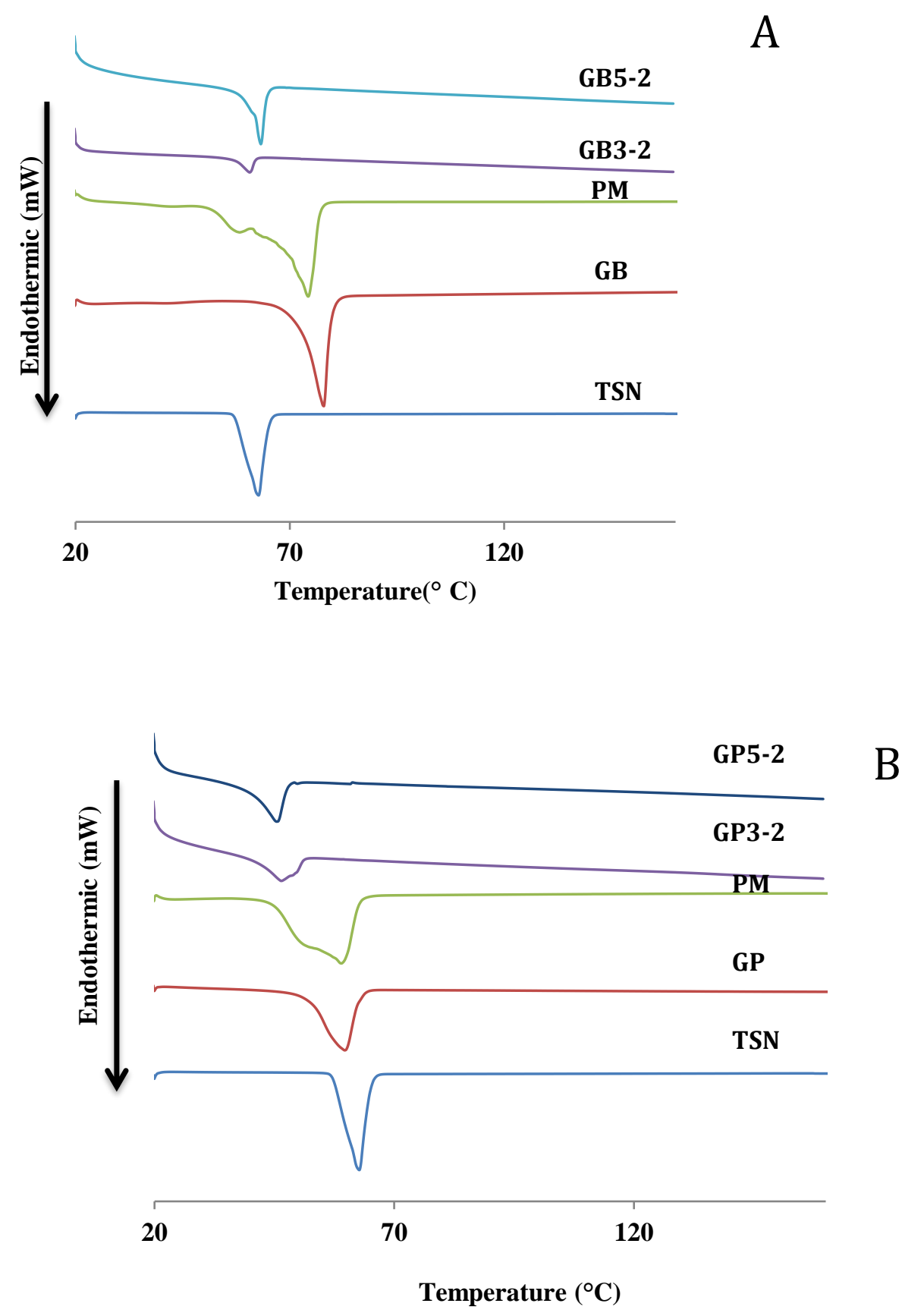

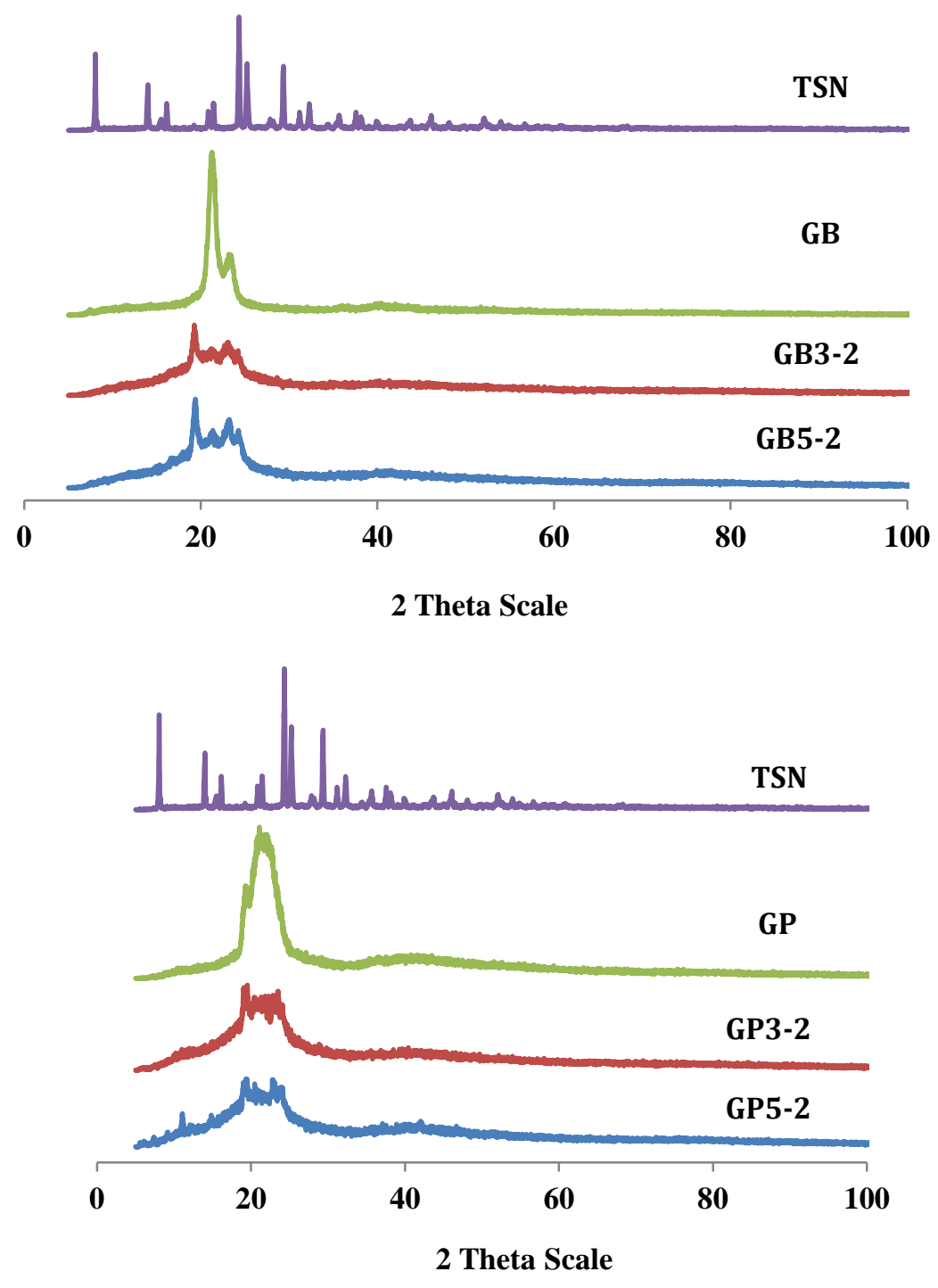

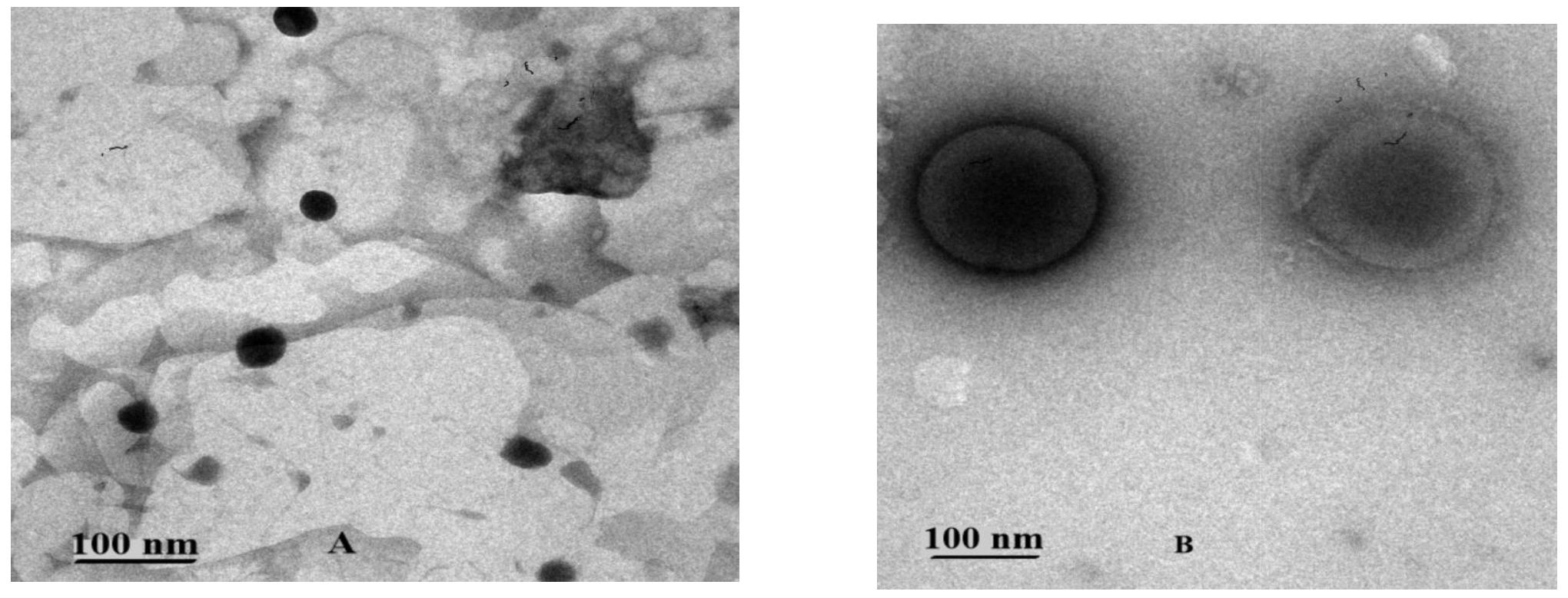

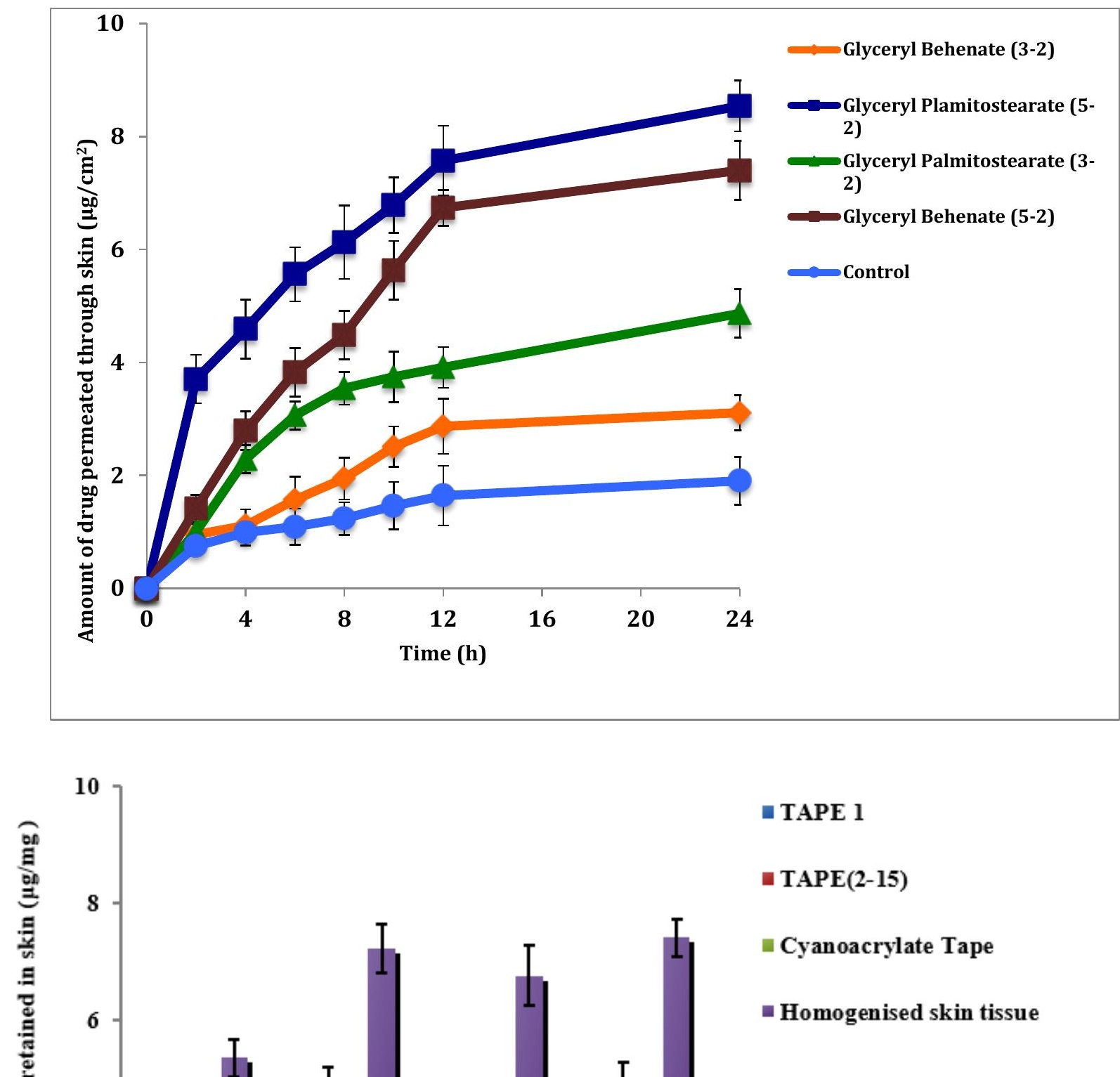

- TAPE 1

TAPE(2-15)

Cyanoacrylate Tape

- Homogenised skin tissue 